\title{
Excess Anxiety's Effect on the Occurrence of Insomnia in Adolescents in Late Adolescence
}

\author{
Bianca Locsin ${ }^{1}$, Maine Salvador ${ }^{1}$ \\ ${ }^{1}$ Manila Central University, Philippines
}

Received: June 15, 2021

Received in Revised: July 14, 2021

Accepted: July 28, 2021

\begin{abstract}
Due to the difficult process of social transition associated with the end of adolescence, this may create complications. For example, the issue of anti-social conduct in teenagers, as well as disagreements with parents, often result in conflict, ties of solidarity that may result in hazardous hazards and emotional illnesses such as stress, excessive anxiety, and depression. Anxiety disorder is a kind of emotional illness that may develop in late adolescence as a result of psychosocial causes. In which late adolescents react to stresses in an inappropriate and accurate manner. Anxiety disorders may impair the development of teaching and learning, since they lead people to suffer information processing distortion. The purpose of this study was to evaluate the impact of excessive worry on the prevalence of sleeplessness in late teenage adolescents. The researchers will conduct an analytical observational study using a cross sectional design. Teenagers with anxiety were classified as having no anxiety by 71 individuals ( 88.8 percent), mild anxiety by 8 people (10.0 percent), and moderate anxiety by one person (1.3 percent). According to the classification of insomnia, there were 48 individuals (60.0 percent) in the severe group, 18 teenagers ( 22.5 percent) in the very severe category, and 14 adolescents (17.5 percent) in the moderate category. There is a link between anxiety and the prevalence of sleeplessness in late teenage teens.
\end{abstract}

Keywords: Insomnia, Anxiety, Adolescent

\section{Introduction}

Humans grow and develop both before and after birth. Physical maturation occurs when the body's muscular tissue, neurological system, and organ function systems become more sophisticated, suggesting that the body is growing. Additionally, development may be defined as "the changes that occur in people or organisms as they approach their level of maturity or adulthood that occur in a systematic, gradual, and continuous manner both physically (physical) and psychically (spiritually)".

Toddlerhood lasts between 0 and 5 years, whereas childhood lasts between 5 and 11 years. Early adolescence lasts from 12 and 16 years. Late adolescence lasts from 17 and 25 years. Early adulthood lasts from 27 and 35 years. Late adulthood lasts from 36 and 45 years. Early old age is defined as 46-55 years of age. 56-65 years is considered late old age. The elderly are defined as those aged 65 and above.

Late adolescents feel more mature since they have more time to spend with their friends and more opportunities to explore beliefs and lifestyles (Brook et al., 2013). Additionally, they want independence and will be more intellectually challenged by academic assignments. $6 \mathrm{At}$ the conclusion of adolescence, they are expected to acquire a variety of skills. Such is adjusting to a new social life, religious maturity, awareness and responsibility, self-acceptance, economic independence, peer interactions, and, often, emotional maturity. 
Due to the difficult process of social transition associated with the end of adolescence, this may create complications. For example, the issue of anti-social conduct in teenagers, as well as disagreements with parents, often result in conflict, ties of solidarity that may result in hazardous hazards and emotional illnesses such as stress, excessive anxiety, and depression.

From Basic Health's study on anxiety disorders. There are $6 \%$ of individuals aged 15 years and older, or about 14 million people, suffer from emotional problems, as evidenced by the prevalence of anxiety symptoms. The capacity to regulate one's emotions is necessary for remaining calm under duress. 9 If a person is resilient, he or she will utilize their positive skills to manage their emotions, concentrate their attention and conduct, and overcome worry, sorrow, or anger in order to settle issues swiftly and quietly. The inability to regulate emotions as a teenager progresses into early adulthood makes late adolescence seem very weighty (Zebrack et al., 2014). Someone who struggles with emotion regulation is likely to be less successful at problem solving. As a result, the issue cannot be successfully resolved.

Anxiety disorders are a kind of emotional illness that may develop in late adolescence as a result of psychosocial influences (Pittman \& Richmond, 2007). When late adolescents react improperly and accurately to stresses, such as novel environmental circumstances. This inaccuracy of reactions may be attributed to selective attention to unfavorable aspects in the surroundings, information processing distortion, and an excessively pessimistic assessment of one's capacity to deal with the stressor. Anxiety disorders may impair the development of teaching and learning, since they lead people to suffer information processing distortion. This obviously impairs one's capacity to concentrate attention and also impairs memory. As a result, patients will seek therapy to alleviate their worry. Late adolescents often face obligations in a variety of circumstances (Carmichael \& Whittaker, 2007). Certain individuals subjectively evaluate these needs, while others see them as obstacles. while some others see requests as potential sources of conflict. 13 Changes in a person's preferred environment may result in concern, anxiety, fear, and a feeling of insecurity linked with dangers both internal and external, referred to as anxiety. "Anxiety may be classified into four levels: mild anxiety, moderate anxiety, severe anxiety, and extremely severe anxiety (panic)." Each person has a unique degree of anxiety that varies according to how they respond to the triggering circumstance.

Divides anxiety into many subtypes, one of which is objective anxiety, which is concerned with negative events. Neurotic anxiety is anxiety induced by a fear that if one's instincts are not utilized, anarchy would result (Neumann, 2017). Finally, moral anxiety is the dread that develops as a result of guilt for doing something you shouldn't have. If this fear is maintained, it may develop into a psychiatric illness. sensory (physical) somatic signals, cardiovascular signs, respiratory signs, gastrointestinal (digestive) indicators, urogenital signs, autonomic indications, and behavioral/attitude signs. When the body is anxious, the sympathetic nervous system's activity is increased, which may result in alterations in the body's reaction.

Stressors encountered in teenagers nearing maturity not only create excessive anxiety, but also result in a variety of issues, ranging from abnormal eating habits to lack of sleep. Insomnia is the inability to fulfill one's sleep requirements, both qualitatively and quantitatively. Insomnia may interfere with human biological cycles, impairing mood, attention, and memory (Palagini et al., 2019).

According to the National Sleep Foundation (NSF), sleep problems may have a variety of negative consequences for people. When a person is sleep deprived, he or she will think and operate less efficiently, make errors, and have difficulty recalling information. This results in decreased productivity, which may result in accidents. Additionally, employees become more irritated, impatient, restless, and sad. This issue often interferes with job and family 
relationships, to the point that social activities are reduced. Sleep deprivation at work is the leading contributor to reduced productivity, absenteeism, and workplace accidents. 24 Insomnia is often a transient or brief disorder. Sleep problems may be as persistent in certain instances. This is often referred to as a sleep adjustment disorder because to the fact that the condition frequently arises in a situational setting of acute depression, such as starting a new job or shortly before an exam. Sleep problems usually resolve after depression has been treated or the person has completed a course of antidepressant therapy. Ad interim insomnia, on the other hand, often recurs when fresh tensions or stresses in the patient's life occur (Ellsworth, 2012).

\section{Insomnia}

Insomnia is a common sleep disorder that is well-known in the general population. Insomnia is the inability to initiate or maintain sleep (Ohayon \& Paiva, 2005). Typically, patients with insomnia have nonspecific complaints in addition to the insomnia itself. This condition can be either temporary or permanent. Insomnia that is brief or transient is most frequently caused by anxiety. While persistent insomnia is frequently associated with the most common problem, difficulty falling asleep. Additionally, insomnia can be classified into three types based on its duration: transient insomnia, which lasts between two and three days, short-term insomnia, which lasts up to three weeks, and long-term insomnia, which can last for an extended period of time and is typically caused by certain medical or psychiatric conditions.

\section{Etiology}

Insomnia can be caused by the following risk factors. The following is an explanation of the risk factors for insomnia. In the elderly, sleep initiation and maintenance are reported to be difficult. This situation arises as a result of aging-related changes in the brain mechanisms that control sleep duration and quality. Additionally, older adults and younger adults have distinct sleep patterns. Sleep requirements decrease as a person matures. Sleep requirements are nine hours at the age of twelve, eight hours at the age of twenty, seven hours at the age of forty, six and a half hours at the age of sixty, and just six hours at the age of eighty.

Women were shown to have a greater risk of sleeplessness than males. This is believed to be linked indirectly to hormonal variables, specifically when a person experiences a psychological condition and feels worried, tense, or unable to regulate their emotions, this may result in a reduction in the hormone estrogen, which can contribute to the increase in sleep problems.

Insomnia may develop as a result of physical problems such as drug misuse, withdrawal symptoms, painful or uncomfortable conditions, or as a result of mental disorders such as anxiety or depression. The most often reported complaints are difficulties starting and sustaining sleep (Young et al., 2008). Insomnia may also be a result of environmental or social factors. For instance, when someone we care about dies, the room's state deteriorates, as do his own thoughts.

Difficulty sleeping is often shown by a lack of sleep, frequent waking up at night, waking up early in the morning, feeling tired during the day, and not rarely falling asleep for an extended period of time (Chung \& Cheung, 2008; Goldman et al., 2008). This results in a reduction in the quality of a person's sleep. The consequence will be seen in daily life, namely a reduction in quality of life, productivity, and safety, as well as a feeling of weakness, tiredness, and indolence as a result of inadequate sleep.

\section{Diagnosis of Insomnia}

Major insomnia is diagnosed when the predominant symptom is unrefreshing sleep or trouble starting or sustaining sleep for at least one month. As an example, consider the following DSM-

Copyright @ 2021, Journal of Asian Multicultural Research for Medical and Health Science Study, Under the license CC BY-SA 4.0 
IV-TR evaluation criteria. For at least one month, the following complaints are usually controlled: difficulties starting or sustaining sleep, or sleep that is not rejuvenating. Sleep deprivation (or related daytime tiredness) causes clinically substantial distress or impairments in social, vocational, and/or other critical aspects of functioning. The sleep disruption is not limited to those suffering from narcolepsy, sleep disorders linked with breathing, circadian rhythm sleep disorders, or parasomnia.

The problem does not always occur in conjunction with another mental illness (eg, major depressive disorder, generalized anxiety disorder, delirium). Additionally, the disruption is not caused by the direct physiological effects of a substance (e.g., an addictive chemical or medicine) or a general medical condition.

Disruptions that occur at least three times each week over a period of at least one month. There is an obsession with sleep deprivation and an exaggerated concern about the repercussions at night and during the day. Dissatisfaction with the amount and quality of sleep causes moderate to severe pain and impairs social and occupational functioning. The existence of symptoms consistent with other mental illnesses such as sadness, anxiety, or obsessions does not negate the need of assessing insomnia. Comorbidities must be mentioned separately since they need distinct treatment. The "sleep duration" (quantity) criteria was omitted from determining the existence of disturbance, owing to the broad range of individual variation. The length of the disturbance that does not satisfy the preceding requirements (e.g., temporary sleeplessness) may be included in this calculation.

Deconditioning method: in this technique, the patient is instructed to use his bed only for sleeping and not for anything else; if the patient does not fall asleep within five minutes, he or she is instructed to rise and do something else. Occasionally, patients benefit from shifting locations or sleeping quarters.

By using stimulus control treatment, called Stimulus Control Therapy, we can educate people about sleep hygiene. Maintaining a consistent sleep and wake-up time, even on vacations. When you are in bed, put away the television, books, and work. Avoid taking naps. Maintain a regular exercise schedule (3-4 times per week), but avoid exercising in the afternoon if it interferes with later sleep. Eliminate or decrease your intake of alcohol, caffeine, cigarettes, and other sleep-inducing drugs. Perform a calming activity before to retiring to bed. Arrange the bedroom such that it is both pleasant and silent. Patients undergoing cognitive treatment for insomnia often have negative ideas and beliefs about the possible implications of their illness. The primary aim of this treatment is to assist patients in overcoming their erroneous ideas and beliefs. Additionally, it may help alleviate the anxiety associated with sleeplessness.

Sleep restriction (restriction) treatment is based on the premise that restricting one's time in bed may assist improve sleep quality later in life. There are two types of medications used to treat insomnia, benzodiazepines and non-benzodiazepines. In the benzodiazepine class, nitrazepam is suggested at a dosage of 5-10 mg/night, flurazepam at a dose of $15-20 \mathrm{mg} / \mathrm{night}$, and estazolam at a dose of 1-2 mg/night. Meanwhile, zolpidem is a non-benzodiazepam medication with a suggested dosage of 10-20 mg/night. The dosage should be reduced in the elderly and increased gradually to prevent oversedation and drunkenness.

The researcher utilized a questionnaire method with a measuring instrument (instrument) for the Jakarta-Insomnia Biology Psychiatric Study Group to collect data on insomnia in the elderly before and after relaxing. This questionnaire has 11 questions, three on sleep stages, four on the effect of sleep deprivation, and four on signs and symptoms. This questionnaire employs an ordinal scale, in which responses are assigned a value between 1 and 4 . Where the total number may be divided into the following categories: There have been no reports of 
difficulties sleeping: If the score is 11-19, it indicates moderate insomnia; if the score is 20-27, it indicates significant sleep difficulty: If the score is between 28 and 36, and the insomnia is severe: if the score is between 37 and 44 .

In essence, anxiety is a natural emotion that every human being experience. Anxiety has become ingrained in the lives of people of all ages. Anxiety is a general emotion that occurs when a person experiences fear or loses confidence in things that are not readily apparent. Anxiety is a natural response when a person is confronted with a highly stressful circumstance. Anxiety may exist alone or in conjunction with other symptoms, depending on the emotional problems experienced.

Anxiety is a natural reaction to a unique hazardous circumstance; it happens throughout growth, change, new or unusual experiences, and in the search for proof of self and purpose in life. Anxiety is a natural human response. However, excessive anxiety, particularly that which has been classified as a disease, may impair a person's ability to function in life. Anxiety is a subjective emotion, where the mental tension associated with it becomes a general response triggered by an inability to deal with a situation or a sense of insecurity. This irregular sensation is unpleasant and may cause physiological and psychological changes in a person.

Anxiety is a physiological reaction to a perceived or imagined danger. An individual may feel anxiety as a result of future uncertainty, or as a result of their belief that something terrible may occur. Anxiety is a frightening condition caused by a perceived danger to one's health. Anxiety is an emotional condition associated with physiological arousal, characterized by an unpleasant sense of tension and dread of something terrible happening. Anxiety is an irrational dread and discomfort. Anxiety is also a driving factor behind aberrant or disordered conduct. Both are assertions, appearances, and manifestations based on the anxiety defense mechanism.

The conclusion that can be made from some of the aforementioned viewpoints is that anxiety is a sensation of discomfort or dread in a potentially dangerous scenario, which may result in worry due to future uncertainty and the concern that something terrible will happen.

\section{Symptoms}

Anxiety is a stressful condition that occurs when an individual's health is threatened. Individuals who are considered normal sometimes feel anxiety as well; often, there will be physical and even mental symptoms. Physical symptoms include numb fingers, racing heartbeat, chilly chills, dizziness, reduced appetite, sleep deprivation, and chest tightness. Symptoms of a mental nature include fear of being overwhelmed by danger, inability to concentrate your attention, a sense of insecurity, and a desire to flee based on reality.

Anxiety also includes subtle and unpleasant features such as the appearance of dread, caution, or vigilance. The symptoms of anxiety that present may not always line up in each individual. Fear and anxiety are two emotions that serve as indicators of impending danger. Fear occurs when dangers are seen directly, are dependent on the environment, and do not immediately affect people. Meanwhile, anxiety occurs when the threat is internal, subtle, or creates difficulties for the person. Anxiety arises as a result of unconscious emotions that are unrelated to tangible things or real-world circumstances. Anxiety symptoms include the following: Fearlessness that is unseen often results in dread and anxiety. The second indication is when emotions become uncontrollable.

Restlessness, tremors, increased perspiration, shortness of breath, heart beating quicker, and weakness are among physical signs. Behavioral signs include a greater tendency to become detached from something, closed, and quickly frightened. Psychological symptoms. 
Uncomfortable in some situations, overwhelming dread of the future, conviction that terrible things will happen, and inability to concentrate

Anxiety often develops over time and is mainly determined by one's life events. Certain events and circumstances may hasten the onset of anxiety attacks. Many variables contribute to an anxiety response, including the following:

The environment may exacerbate anxiety as a result of negative interactions with family, friends, and coworkers. As a result, an individual may feel uneasy in his or her own surroundings. Anxiety may develop as a consequence of suppressing emotions, being depressed for an extended period of time, and being unable to come up with answers to their feelings.

Anxiety occurs as a result of the interaction of the mind and body, which results in feelings of anxiety under specific circumstances (during pregnancy, adolescence, and while recovering from sickness). Numerous factors contribute to anxiety, including the following: Anxiety and dread that emerge as a result of perceiving the danger that lurks inside his thoughts, Anxiety caused by guilt, Anxiety manifested as illness (anxiety).

Anxiety generated within the family setting, namely the presence of misconceptions and a feeling of parental ignorance toward their children, may make youngsters feel uneasy at home. If someone is in a positive social environment, it will have a positive effect; but, if they are in a negative environment, it will result in negative conduct, as well as a negative judgment in the eyes of others who see it. Physical infirmity may contribute to a person's mental deterioration.

Unfavorable beginning environment. The environment is the primary element that may influence an individual's anxiety; if these circumstances are not favorable, personality development might be harmed. Anxiety is characterized by mood fluctuations, which occur spontaneously in each person. Distinguishes three kinds of anxiety: Rational Anxiety is a dread of immediate threats, such as awaiting test results. A person who feels emotions as a result of unique circumstances is usually not seen as dangerous. Fundamental worry about his identity, his purpose in life, and the direction his life would go in the future. This anxiety is classified as existential and plays a significant part in human existence. Videbeck makes his case by classifying worry into four categories.

When anything is out of sync with a person's daily activity, mild uneasiness develops in his emotions. Sensory stimulation enhances and assists people in focusing their attention on learning, problem solving, thinking, acting, tasting, and self-protection. Minor anxiety manifests physically as mild muscular tension, a little restlessness, but continued concentration; cognitive responses seem calm, confident, have less thoughts of failure, and are more attentive; and emotional reactions manifest as being somewhat unconscious, aroused, and peaceful.

Anxiety occurs when a person perceives something as unsettling, enabling him to be serious about something vital but also limiting his view. Physical responses include mild muscle tension, sweating, pacing, increased alertness and tension, frequent urination, headaches, changes in sleep patterns, and back pain; Cognitive responses include an emphasis on the higher the stimulus, the greater the range of problem solving, and the use of focus; and Emotional responses include being uncomfortable, irritable, and becoming impatient.

Severe anxiety causes a person to be solemn and unable to focus about anything else. At this point, the person begins to see worry as a danger to himself. Severe muscle tension, hyperventilation, poor eye contact, increased sweating, rapid speech, high pitched voice, aimless and reckless actions, jaw tension, gritting teeth, pacing, shouting, squeezing hands, and 
shaking are all physical responses; cognitive responses include difficulty thinking due to a broken concentration level, inability to solve problems, and inability to consider information.

Panic is a feeling of losing control, of being unable to accomplish anything while being given instructions. Panic disorder is characterized by personality disintegration and may be fatal. Increased muscular activity, altered perception, and a lack of logical thinking are all symptoms of panic. Physical responses to panic include extreme muscle tension, gross motor agitation, inability to sleep, a sense of stress, and decreased neurotransmitters; Panic cognitive responses include illogical thoughts as a result of narrow perception, disturbed, chaotic personality, inability to solve problems, and irrational thinking; and even hallucinations, delusions, and delusions can occur.

\section{The association between excessive anxiety and sleeplessness in teenagers}

The findings of study suggest that there is a connection between anxiety and sleeplessness in late adolescence teenagers. The Chi Square test demonstrates that the significance value of 0.001 is less than 0.05 . Insomnia is the inability to fulfill one's sleep requirements, both qualitatively and quantitatively. Insomnia may interfere with human biological cycles, impairing emotions, attention, and memory.

Anxiety stems from an unconscious emotion inside one's own psyche and is unrelated to actual things or circumstances. Excessive anxiety in students causes the student to think too hard, making it difficult to regulate his emotions, which results in increased tension and trouble falling asleep. When the body is anxious, the sympathetic nervous system's function is increased, resulting in alterations in the body's reaction. Stressors encountered in teenagers nearing maturity not only create excessive anxiety, but also result in a variety of issues, ranging from abnormal eating habits to lack of sleep.

Insomnia is often a transient or brief disorder. However, in certain instances, insomnia may develop into a chronic condition. It is often referred to as sleep adjustment disorder since it is most frequently associated with acute depression in a situational setting, such as a new job or an exam. Insomnia typically subsides after the depression has passed or the person has acclimated to it. However, transitory insomnia often recurs when the patient's life is confronted with new or comparable stressors.

This study has limitations, in that the researcher is restricted to studying one component associated with the incidence of insomnia, obviating the possibility of determining additional variables that contribute to insomnia. Because the data input procedure is not aided by an assistant, it takes an inordinate amount of time.

\section{Conclusion}

The following conclusions may be made from the findings of the research entitled The connection between excessive anxiety and the prevalence of teenage insomnia. There is a correlation between anxiety and sleeplessness in late teenage teens. The chi square significance value of 0.001 , which is less than 0.05 , demonstrates this. The findings of this research show that there is a significant connection between anxiety and the prevalence of sleeplessness in late teenage teens.

\section{References}

Brook, J. S., Lee, J. Y., Finch, S. J., Seltzer, N., \& Brook, D. W. (2013). Adult work commitment, financial stability, and social environment as related to trajectories of marijuana use beginning in adolescence. Substance abuse, 34(3), 298-305. 
Zebrack, B., Kent, E. E., Keegan, T. H., Kato, I., Smith, A. W., \& Aya Hope Study Collaborative Group 1. (2014). "Cancer sucks," and other ponderings by adolescent and young adult cancer survivors. Journal of psychosocial oncology, 32(1), 1-15.

Pittman, L. D., \& Richmond, A. (2007). Academic and psychological functioning in late adolescence: The importance of school belonging. The Journal of Experimental Education, 75(4), 270-290.

Carmichael, G. A., \& Whittaker, A. (2007). Choice and circumstance: Qualitative insights into contemporary childlessness in Australia. European Journal of Population/Revue europeenne de demographie, 23(2), 111-143.

Neumann, F. L. (2017). Anxiety and politics. tripleC: Communication, Capitalism \& Critique. Open Access Journal for a Global Sustainable Information Society, 15(2), 612-636.

Palagini, L., Bastien, C. H., Marazziti, D., Ellis, J. G., \& Riemann, D. (2019). The key role of insomnia and sleep loss in the dysregulation of multiple systems involved in mood disorders: A proposed model. Journal of sleep research, 28(6), e12841.

Ellsworth, D. L. (2012). Stress Therapy Empowering Prevention (STEP): A Healthy Lifestyle Program for Breast Cancer Patients. Integrative Cardiac Health Project, 45.

Ohayon, M. M., \& Paiva, T. (2005). Global sleep dissatisfaction for the assessment of insomnia severity in the general population of Portugal. Sleep Medicine, 6(5), 435-441.

Young, J. S., Bourgeois, J. A., Hilty, D. M., \& Hardin, K. A. (2008). Sleep in hospitalized medical patients, part 1: factors affecting sleep. Journal of hospital medicine: an official publication of the Society of Hospital Medicine, 3(6), 473-482.

Chung, K. F., \& Cheung, M. M. (2008). Sleep-wake patterns and sleep disturbance among Hong Kong Chinese adolescents. Sleep, 31(2), 185-194.

Goldman, S. E., Ancoli-Israel, S., Boudreau, R., Cauley, J. A., Hall, M., Stone, K. L., ... \& Newman, A. B. (2008). Sleep problems and associated daytime fatigue in communitydwelling older individuals. The Journals of Gerontology Series A: Biological Sciences and Medical Sciences, 63(10), 1069-1075. 